\title{
Influence of both chewing and lubrication during oral processing of hydrogels on hunger and energy intake
}

\author{
E.M. Krop ${ }^{1}$, M.M. Hetherington ${ }^{2}$, S. Miquel ${ }^{3}$ and A. Sarkar ${ }^{1}$ \\ ${ }^{1}$ Food Colloids and Processing group, School of Food Science and Nutrition, University of Leeds, LS2 9JT, UK, \\ ${ }^{2}$ School of Psychology, University of Leeds, LS2 9JT, UK and ${ }^{3}$ Mars Wrigley Confectionery, 1132 West Blackhawk \\ Street, Chicago, IL 60642, USA.
}

It is widely accepted that oral processing is an important factor in the regulation of food intake, with slow eating rate, high number of chews, longer oro-sensory exposure, smaller bites and harder food textures being recommended for decreasing the energy intake ${ }^{(1)}$. However, the effects of oral lubrication remain unclear. This study aimed to determine the individual and combined effects of the chewing and lubrication aspects of oral processing on subjective appetite and snack intake. Model biopolymer gels with mint flavouring were designed varying in their textural properties (as measured instrumentally and sensorially, unpublished data) to generate different oral processing behaviour as related to the chewing and the lubrication.

Fifty-five participants $\left(26.3 \pm 7.1\right.$ years old, BMI $\left.23.0 \pm 3.2 \mathrm{~kg} / \mathrm{m}^{2}\right)$ were recruited to participate in a between-subjects randomized study design with three hydrogel samples varying in their chewing and lubrication attributes, as well as a control group. For the control group, a mint tea with the same green food colouring as in the gels was used. First, participants received a sandwich lunch, followed 3 hours later by the mint preload and an ad libitum salty snack. Hunger, fullness, desire to eat and appetite were rated on $100 \mathrm{~mm}$ visual analogue scales (VAS) before $\left(\mathrm{t}_{0}\right)$ and after the preload $\left(\mathrm{t}_{1}\right)$, as well as after the snack $\left(\mathrm{t}_{3}\right)$, and the snack intake was weighed.

Snack intake appeared to be suppressed after eating the low chewing/high lubricating sample as compared to the high chewing/low lubricating, medium chewing/high lubricating sample and the tea (reduced by $30 \%$ ). No other significant differences were found for food intake $(p>0.05)$. Hunger ratings decreased from $t_{0}$ to $t_{3}(p<0.05)$, however differences between conditions were rather subtle. Thus, a model food with high oral lubrication properties could help reduce the snack intake, but more research is necessary on the interaction between chewing and lubrication properties.

1. Krop EM, Hetherington MM, Nekitsing C et al. (2018) Influence of oral processing on appetite and food intake - A systematic review and meta-analysis. Appetite 125, 253-69. 